\title{
Thermodynamics of a higher-order topological insulator
}

\author{
R. Arouca $\odot,{ }^{1,2, *}$ S. N. Kempkes, ${ }^{1, \dagger}$ and C. Morais Smith ${ }^{1, \ddagger}$ \\ ${ }^{1}$ Institute for Theoretical Physics, Center for Extreme Matter and Emergent Phenomena, Utrecht University, Princetonplein 5 , \\ 3584 CC Utrecht, The Netherlands \\ ${ }^{2}$ Instituto de Física, Universidade Federal do Rio de Janeiro, C.P. 68528, Rio de Janeiro, RJ 21941-972, Brazil
}

(Received 19 December 2019; accepted 25 March 2020; published 29 April 2020)

\begin{abstract}
We investigate the order of the topological quantum phase transition in a two-dimensional quadrupolar topological insulator within a thermodynamic approach. Using numerical methods, we separate the bulk, edge, and corner contributions to the grand potential and detect different phase transitions in the topological phase diagram. The transitions from the quadrupolar to the trivial or to the dipolar phase are well captured by the thermodynamic potential. On the other hand, we have to resort to a grand potential based on the Wannier bands to describe the transition from the trivial to the dipolar phase. The critical exponents and the order of the phase transitions are determined and discussed in the light of the Josephson hyperscaling relation.
\end{abstract}

DOI: 10.1103/PhysRevResearch.2.023097

\section{INTRODUCTION}

Topological states of matter have attracted a great deal of attention during the last decade. In addition to the usual discrete symmetries that compose the 10-fold way [1-3], the periodic table of topological materials was extended by the inclusion of crystalline symmetries [4,5], which led to the prediction of new kinds of topological phases. A typical example is the recent proposal of higher-order topological insulators (HOTIs) [6].

HOTIs, or more generally higher-order topological phases, are such that the symmetry protected modes (SPMs) associated with the boundary between the topological phase and the vacuum occur in the system not with codimension 1 , but with codimension 2 or higher. This means that for two-dimensional (2D) systems, these modes will appear at the OD corners. Similarly, for 3D systems they do not arise at the 2D surface but at the $1 \mathrm{D}$ hinges or at the $0 \mathrm{D}$ corners of the system. The existence of HOTIs was experimentally verified in a variety of systems, such as bismuth [7], electrical circuits [8,9], and acoustic [10,11] and electronic [12] systems, and new types of HOTIs and higher-order topological superconductors were recently proposed [13-16].

A very interesting question posed by this kind of topological phase is the fate of the bulk-boundary correspondence $[17,18]$. A thermodynamic analysis of topological quantum phase transitions (TQPTs) [19-22] inspired by Hill's thermodynamics [23-27] has been successful in describing the

\footnotetext{
*r.aroucadealbuquerque@uu.nl

†s.n.kempkes@uu.nl

‡c.demoraissmith@uu.nl
}

Published by the American Physical Society under the terms of the Creative Commons Attribution 4.0 International license. Further distribution of this work must maintain attribution to the author(s) and the published article's title, journal citation, and DOI. critical behavior of several models and recently this method was used to devise topological heat machines [28]. Here, we extend this thermodynamical approach to describe HOTIs, i.e., to deal with systems that have both edge and corner modes. For this, we investigate the model proposed in Ref. [6], which is a 2D Su-Schrieffer-Heeger (SSH) model, with broken symmetry between the $x$ and the $y$ directions and different intra- and intercell hopping parameters. In addition, a $\pi$ flux pierces the plaquettes, such that some of the hopping parameters are negative. This model exhibits a rich phase diagram, with phases characterized by the polarization: depending on the ratio between the different hopping parameters, the system is in a quadrupolar, a dipolar, or a trivial phase. To describe the phase transitions, we calculate the correlation functions for the bulk, edge, and corner contributions and look for divergences or jumps in their derivatives, which signal a phase transition.

For the transition from the trivial to the quadrupolar state, we find that the bulk exhibits a third-order, the edge a second-order transition, and the corner a first-order phase transition. The transition from the dipolar to the quadrupolar phase is also visible in the thermodynamic response at the edge and corner contributions, although it is invisible for the bulk. Finally, the transition from the trivial to the dipolar phase cannot be captured by the thermodynamic potential description, since there is no bandgap closing at the transition. To characterize the topology of these phases, the Wannier spectrum was investigated [6]. The gap in the spectrum of the Wannier centers closes for all phase transitions, and therefore we extracted an effective grand potential from the Wannier spectrum. We found that the Wannier grand potential exhibits discontinuities at all phase transitions, fully characterizing the critical behavior of the system. The critical exponents were extracted by analyzing the functional dependence of the closing of either the bandgap or the Wannier gap, and the order of the phase transitions was determined using the Josephson hyperscaling relation. An excellent agreement is found with the order obtained by analyzing the discontinuities in the thermodynamic or the Wannier grand potential. 
The outline of the paper is as follows: In Sec. II, we present the quadrupolar topological insulator model proposed in Ref. [6], focusing specifically on the different phases of the phase diagram. Next, in Sec. III, we extend the thermodynamical description of topological materials to deal with systems composed of bulk, edge, and corner contributions and explain how one can devise a subtraction scheme to identify the critical behavior of each part. In Sec. IV, we apply this approach to investigate the quadrupolar-to-trivial phase transition and show how the order of the phase transition can be understood by determining the critical exponents and using Josephson's hyperscaling relation. In Sec. V, we perform the same analysis to other phase transitions in the phase diagram and show that the procedure does not apply due to the absence of a gap closing in some of the topological phase transitions. In Sec. VI, we show that a grand potential calculated from the Wilson loop spectra, which we name the Wannier grand potential, is sensitive to all phase transitions, but with critical exponents of a system with smaller dimensions, which we identify to be the edge of the original lattice.

\section{QUADRUPOLAR TOPOLOGICAL INSULATOR}

The existence of HOTIs was proposed based on the modern theory of polarization [6,29-31], which associates the projected position operator with the Wilson loop and a topological index. In these systems, there are SPMs that are localized not over the entire surface of the material, but on parts of this surface. These modes are analogous to multipoles in classical electrodynamics. To realize a quadrupolar topological insulator in a $2 \mathrm{D}$ lattice, such that the protected modes are localized at the corners of the lattice, Benalcazar et al. [6] proposed a model which, in the presence of $C_{4}$ symmetry, has a vanishing dipolar order and a nonzero charge localized in the corner due to the bulk quadrupolar moment [18].

The Hamiltonian of this model reads

$$
\begin{aligned}
H= & \sum_{\mathbf{R}} \gamma_{x}\left[c_{\mathbf{R}, 1}^{\dagger} c_{\mathbf{R}, 3}+c_{\mathbf{R}, 2}^{\dagger} c_{\mathbf{R}, 4}+\text { H.c. }\right] \\
& +\gamma_{y}\left[c_{\mathbf{R}, 1}^{\dagger} c_{\mathbf{R}, 4}-c_{\mathbf{R}, 2}^{\dagger} c_{\mathbf{R}, 3}+\text { H.c. }\right] \\
& +\lambda_{x}\left[c_{\mathbf{R}, 1}^{\dagger} c_{\mathbf{R}+\mathbf{x}, 3}+c_{\mathbf{R}+\mathbf{x}, 2}^{\dagger} c_{\mathbf{R}, 4}+\text { H.c. }\right] \\
& +\lambda_{y}\left[c_{\mathbf{R}, 1}^{\dagger} c_{\mathbf{R}+\mathbf{y}, 4}-c_{\mathbf{R}, 3}^{\dagger} c_{\mathbf{R}+\mathbf{y}, 2}+\text { H.c. }\right],
\end{aligned}
$$

where $c_{\mathbf{R}, i}^{(\dagger)}$ denotes the destruction (creation) operator at site $i$ in the unit cell labeled by $\mathbf{R}$ (see sketch in the inset in Fig. 1), and $\gamma_{x, y}\left(\lambda_{x, y}\right)$ are the intracell (intercell) hopping parameters in the $x$ or $y$ direction, respectively. Note the negative hopping terms in Eq. (1), which correspond to a $\pi$ flux (gauge field) piercing the plaquettes. The model exhibits a quadrupolar phase transition when the hopping parameters are $\gamma_{x}=\gamma_{y}=$ $\gamma, \lambda_{x}=\lambda_{y}=1$, and $|\gamma|<1$, such that the different corners are related by $C_{4}$ symmetry and the $\pi$ flux in the hopping cancels the edge currents.

The phase diagram of this system is shown in Fig. 1 [6]. The topological index that characterizes the topological phase is $\mathbf{p}=\left(p_{x}^{\zeta_{y}^{-}}, p_{y}^{\zeta_{x}^{-}}\right)$, where $p_{x / y / x}^{\zeta_{y}^{-}}$is the $x / y$ polarization of the Wannier band $\zeta_{x / y}\left(k_{y} / k_{x}\right)$ localized below the gap. This polarization is quantized in units of $1 / 2$ and is defined

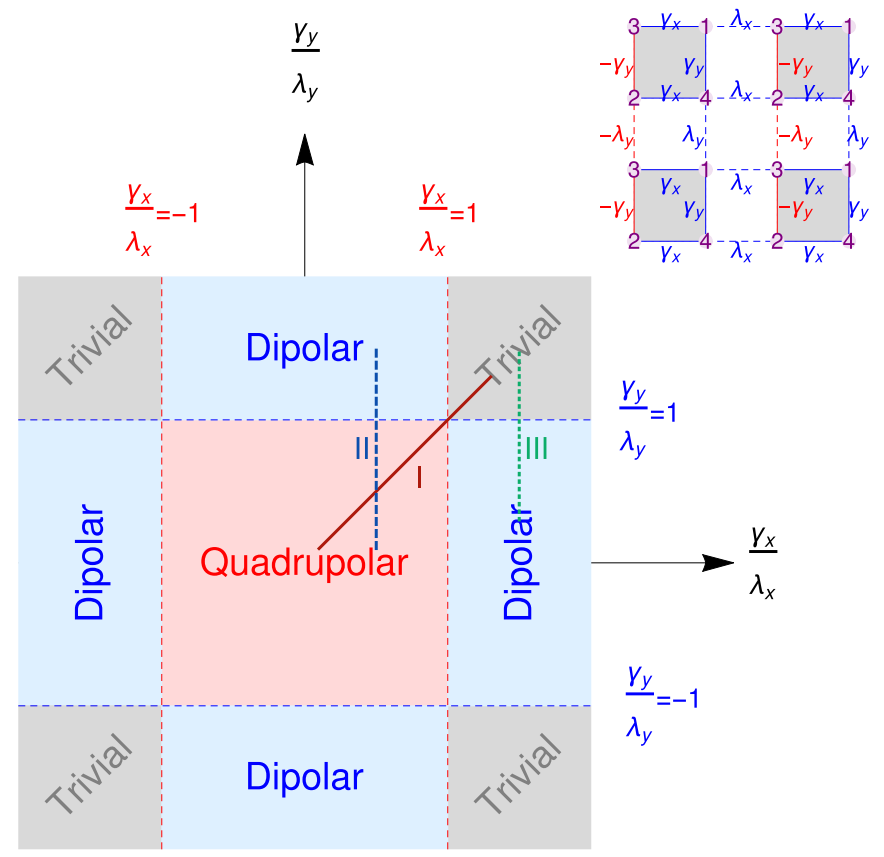

FIG. 1. Phase diagram of Hamiltonian (1) showing the topological phases. For $\left|\gamma_{x} / \lambda_{x}\right| \leqslant 1$ and $\left|\gamma_{y} / \lambda_{y}\right| \leqslant 1$ the system is in the quadrupolar phase. The dipolar phases are the ones where only one of the ratios $\left|\gamma_{x} / \lambda_{x}\right|$ or $\left|\gamma_{y} / \lambda_{y}\right|$ is smaller than 1 ; when both are larger than 1 , the system is in the trivial state. We consider in the following sections the transitions from the trivial to the quadrupolar state (path I; red), the dipolar to the quadrupolar state (path II; blue), and the trivial to the dipolar state (path III; green). Inset: Schematic of the lattice for Hamiltonian (1). The intercell hopping parameters $\lambda$ are represented by dashed lines, while the intracell hopping parameters $\gamma$ are represented by solid lines. Negative values of hopping, created by a $\pi$ flux, are represented by red links.

$\bmod 1$, such that it is either 0 or $1 / 2$, characterizing a $\mathbb{Z}_{2} \times$ $\mathbb{Z}_{2}$ invariant. The trivial phase has $\mathbf{p}=(0,0)$, the dipolar phase has $\mathbf{p}=(1 / 2,0)$ or $\mathbf{p}=(0,1 / 2)$, and the quadrupolar phase has $\mathbf{p}=(1 / 2,1 / 2)$. As the polarization is calculated from the Wannier bands related to the bulk spectrum, the appearance of zero modes in the corner is related to a gap closing in the bulk, showing a new kind of bulk-boundary correspondence $[17,18]$.

\section{THERMODYNAMICS OF THE QUADRUPOLAR PHASE TRANSITION}

TQPTs are QPTs and therefore one should in principle be able to describe their thermodynamic behavior. However, the Ginzburg-Landau scheme fails to characterize them because they do not have a local order parameter. In addition, taking the thermodynamic limit is also a problem because one is interested in the edges of the system and these do not contribute in this limit anymore. Nevertheless, a scaling analysis can be used [32-40], and particularly, it was shown in previous studies [19-22] that a generalized Ehrenfest's classification [41,42] still holds for describing topological phase transitions and allows their characterization by the order of discontinuity or divergence of the derivative of the grand potential. 
The bulk-boundary correspondence relates the nontrivial topology of the bulk, which is associated with a winding number, to the presence of SPMs at the boundary. In this way, the phase transition in the bulk (signaled by the gap closing) is connected to the one at the boundaries (signaled by the appearance/disappearance of the SPMs). However, the bulk and the boundary scale differently with the size of the system. Therefore, a thermodynamic description of TQPTs is more subtle. This leads to a nonextensive grand potential, as only the part of the grand potential related to the bulk scales with the number of particles in the system.

The solution to this conundrum is provided by an approach inspired by Hill thermodynamics [19-28,43-45]. Within this method, the system is divided into many subsets and the thermodynamic identity is derived without the assumption of extensivity of the energy. Hence, one can describe a finitesize system, for which the bulk, the edge, and the corner contributions scale differently with the system size. For the 2D system under investigation, we extended this analysis to deal with systems for which the bulk scales with $L^{2}$, the edge with $L^{1}$, and the corner with $L^{0}$. The grand potential then can be written as

$$
\begin{aligned}
\Omega & =\Omega_{\text {bulk }}+\Omega_{\text {edge }}+\Omega_{\text {corner }} \\
& =\omega_{\text {bulk }} L^{2}+\omega_{\text {edge }} L+\omega_{\text {corner }},
\end{aligned}
$$

where the $\omega$ 's are the intensive contributions for each component of the system. The scaling of the different $\omega$ values reported in Appendix A confirms that indeed they are intensive quantities.

For Hamiltonian (1), we can write $\Omega$ as

$$
\Omega=-\frac{1}{\beta} \ln \left\{\operatorname{Tr}\left[e^{-\beta(\hat{H}-\mu \hat{N})}\right]\right\},
$$

with $\hat{N}$ denoting the number operator, $\mu$ the chemical potential, and $\beta=1 / k_{B} T$. One can then perform the calculation for different system sizes and separate the components in Eq. (2), by verifying how $\Omega$ scales with the system size $[19,20]$.

However, there is another scheme to obtain these contributions using a more geometrical approach [22]. Depending on the boundary conditions of the system, some of these terms are not present, as suggested from the different spectra in Fig. 2. For instance, if we use periodic boundary conditions, there is no boundary between the system and a trivial vacuum. Hence, there is neither a corner nor an edge contribution to the grand potential. Similarly, if we use periodic boundary conditions only along the $x$ or $y$ direction, the system will have no corner contribution, but just one of the edge contributions. Therefore, we can isolate the different terms in Eq. (2) by subtracting the grand potential of systems with different boundary conditions. We represent pictorially the process of subtraction in Fig. 3.

\section{A. Correlation functions in thermodynamics}

Instead of dealing with the grand potential, we consider its derivative. The derivative is related to correlation functions, which will allow us to identify the phase transition. As a matter of fact, if the control parameter of the phase transition
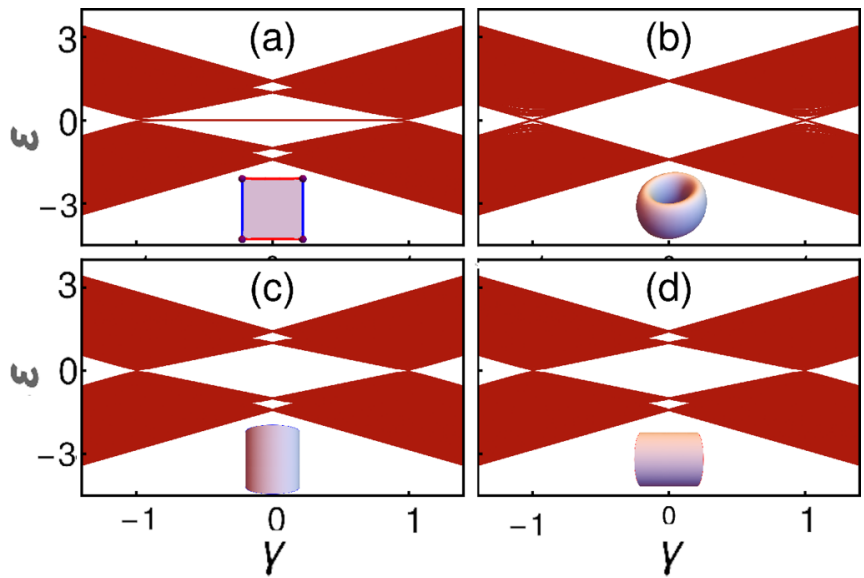

FIG. 2. Spectrum of Hamiltonian (1) for (a) open, (b) fully periodic, (c) $x$-periodic, and (d) $y$-periodic boundary conditions. We fix $\lambda_{x}=\lambda_{y}=1$ and vary the intracell hopping parameters as $\gamma_{x}=\gamma_{y}=\gamma$ (path I in Fig. 1) for a lattice with $40 \times 40$ unit cells. The TQPT occurs for $|\gamma|=1$, at which there is a gap closing and the emergence/disappearance of zero-energy modes. The zero modes are present only with open boundary conditions, confirming that indeed these modes are due to corner states. The similarity between the $x$-periodic and the $y$-periodic spectra is due to the $C_{4}$ symmetry of Hamiltonian (1) for the chosen parameter values.

is $\eta$, the derivative of $\Omega$ with respect to $\eta$ in Eq. (3) is

$$
\begin{aligned}
\frac{\partial \Omega}{\partial \eta} & =\frac{1}{\mathcal{Z}} \operatorname{Tr}\left\{\left[\frac{\partial \hat{H}}{\partial \eta}-\frac{\partial \mu}{\partial \eta} \hat{N}\right] e^{-\beta(\hat{H}-\mu \hat{N})}\right\} \\
& =\left\langle\frac{\partial \hat{H}}{\partial \eta}-\frac{\partial \mu}{\partial \eta} \hat{N}\right\rangle,
\end{aligned}
$$

(a)

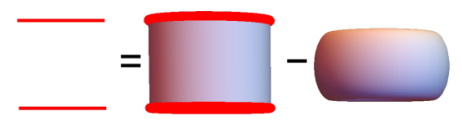

(b)

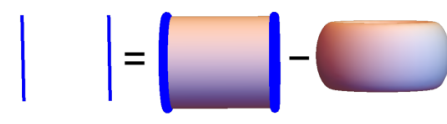

(c)

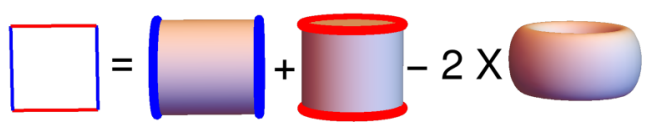

(d)

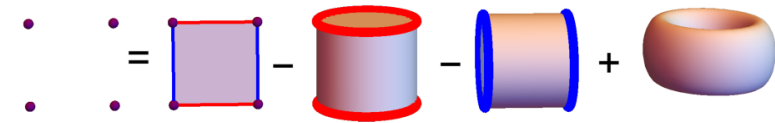

FIG. 3. Representation of the subtraction schemes used to obtain the boundary contributions to the grand potential. (a) Scheme to obtain the $y$-edge contribution from the $x$-periodic and fully periodic boundary condition systems; (b) similarly for the $x$-edge contribution. (c) Scheme to obtain the complete edge contribution from the $y$-periodic, $x$-periodic, and fully periodic boundary condition systems; (d) scheme to obtain the corner contribution from the open, $x$-periodic, $y$-periodic, and fully periodic boundary condition systems. 
and this is equal to a correlation function for a Hamiltonian in second quantization. Hence, the discontinuity in $\Omega$ is encoded in this correlation function [22].

From the set of eigenstates, we can obtain the correlation functions using a matrix $\mathbb{S}$ that relates a vector composed of the eigenstates $\boldsymbol{\Psi}$ and a vector composed of the annihilation operators $\mathbf{C}$,

$$
\begin{aligned}
& \boldsymbol{\Psi}=\mathbb{S}^{-1} \mathbf{C}, \\
& \mathbf{C}=\mathbb{S} \boldsymbol{\Psi},
\end{aligned}
$$

and we can write a correlation function for the $c$ operators as

$$
\left\langle c_{\rho}^{\dagger} c_{\sigma}\right\rangle(T, \mu)=\sum_{m, n} S_{\rho, m}^{*} S_{\sigma, n}\left\langle\psi_{m}^{\dagger} \psi_{n}\right\rangle(T, \mu),
$$

where $\rho$ and $\sigma$ are labels for the $c$ operators and $m$ and $n$ for the energy states. As $\psi^{\dagger}$ is the creation operator of the eigenstates of the Hamiltonian, the correlation function $\left\langle\psi_{m}^{\dagger} \psi_{n}\right\rangle(T, \mu)$ will be simply the occupation of each energy level, given by the Fermi-Dirac distribution multiplied by $\delta_{m, n}$ :

$$
\left\langle c_{\rho}^{\dagger} c_{\sigma}\right\rangle(T, \mu)=\sum_{m} S_{\rho, m}^{*} S_{\sigma, m} \frac{1}{e^{\beta\left(\varepsilon_{m}-\mu\right)}+1} .
$$

Hence, by knowing the spectrum of the system, one can calculate the correlation functions. In particular, for the quantum phase transition under consideration, we evaluate the correlation function for $T=0$ and $\mu=0$. In this case, the Fermi-Dirac distribution is a step function, which vanishes for $\varepsilon>\varepsilon_{F}=0$, and Eq. (7) reduces to

$$
\left\langle c_{\rho}^{\dagger} c_{\sigma}\right\rangle(T=0, \mu=0)=\sum_{\varepsilon_{m} \leqslant 0} S_{\rho, m}^{*} S_{\sigma, m} .
$$

In this way, the correlation functions can be obtained by diagonalizing $\hat{H}$. Note that for different boundary conditions, the spectrum of the system and the wave functions can be different, which ultimately lead to different correlation functions.

\section{B. Correlation functions for the quadrupolar transition}

To describe the thermodynamic response of a system divided into bulk, edge, and corner, we take the derivatives of each term in Eq. (2). For Hamiltonian (1), we write the derivative of $\Omega$ with respect to $\gamma_{x}=\gamma_{y}=\gamma$ given by Eq. (4) as

$$
\begin{aligned}
\frac{\partial \Omega}{\partial \gamma}= & \sum_{\mathbf{R}}\left\langle c_{\mathbf{R}, 1}^{\dagger}\left(c_{\mathbf{R}, 3}+c_{\mathbf{R}, 4}\right)+\text { H.c. }\right\rangle \\
& +\left\langle c_{\mathbf{R}, 2}^{\dagger}\left(c_{\mathbf{R}, 4}-c_{\mathbf{R}, 3}\right)+\text { H.c. }\right\rangle \\
= & \left\langle C^{\dagger} C\right\rangle,
\end{aligned}
$$

where in the last line we have introduced the notation $\left\langle C^{\dagger} C\right\rangle$ for the whole correlation function.

To obtain the terms of Eq. (2), we identify the different contributions for each boundary condition following the scheme in Fig. 3. The scheme follows from the scaling analysis in Eq. (2). For a fully periodic system, the correlation function is the same for each unit cell. Hence, the total correlation function is calculated by multiplying the one for an individual unit cell by the number of unit cells. This contribution scales with $L^{d}$, where $d$ is the dimension. When considering semiperiodic boundary conditions, the same argument holds for a supercell along the open boundary and the correlation function scales with $L^{d-1}$. Since the spectrum is the same for the periodic and semiperiodic boundary conditions, apart from the boundary modes, we obtain the contribution of these boundary modes when subtracting the two contributions. This means we can assume that the contributions coming from this system contain only $\Omega_{\text {bulk }}$, such that

$$
\frac{\partial \Omega_{\text {bulk }}}{\partial \gamma}=\left\langle C^{\dagger} C\right\rangle^{\text {per }}=L^{2} \frac{\partial \omega_{\text {bulk }}}{\partial \gamma},
$$

where the upper index "per" denotes that the correlation function was calculated using periodic boundary conditions. As the system is periodic, the above correlation function does not depend on the position and the sum will be just $L^{2}$ times the correlation function for some point that gives us $\omega_{\text {bulk }}$.

Then, if we subtract the correlation functions associated with the system with fully periodic boundary conditions from those associated with the system with open boundary conditions,

$$
\begin{aligned}
\frac{\partial \Omega}{\partial \gamma}-\frac{\partial \Omega_{\text {bulk }}}{\partial \gamma} & =\left\langle C^{\dagger} C\right\rangle^{\text {open }}-\left\langle C^{\dagger} C\right\rangle^{\text {per }} \\
& =\frac{\partial \Omega_{\text {edge }}}{\partial \gamma}+\frac{\partial \Omega_{\text {corner }}}{\partial \gamma},
\end{aligned}
$$

we obtain only the edge and corner contributions to the grand potential.

Next, we assume that the $x$-periodic system has only the $y$-edge and the bulk contributions,

$$
\frac{\partial \Omega_{y \text { edge }}}{\partial \gamma}+\frac{\partial \Omega_{\text {bulk }}}{\partial \gamma}=\left\langle C^{\dagger} C\right\rangle^{x \text {-per }},
$$

and similarly for the $y$-periodic system,

$$
\frac{\partial \Omega_{x \text { edge }}}{\partial \gamma}+\frac{\partial \Omega_{\text {bulk }}}{\partial \gamma}=\left\langle C^{\dagger} C\right\rangle^{y-\text { per }} .
$$

Therefore, the edge term corresponding to the sum of $\Omega_{x \text { edge }}+\Omega_{y \text { edge }}$ will be

$$
\begin{aligned}
\frac{\partial \Omega_{\text {edge }}}{\partial \gamma} & =\frac{\partial \Omega_{y \text { edge }}}{\partial \gamma}+\frac{\partial \Omega_{x \text { edge }}}{\partial \gamma} \\
& =\left\langle C^{\dagger} C\right\rangle^{y \text {-per }}+\left\langle C^{\dagger} C\right\rangle^{x \text {-per }}-2\left\langle C^{\dagger} C\right\rangle^{\text {per }} \\
& =L \frac{\partial \omega_{\text {edge }}}{\partial \gamma} .
\end{aligned}
$$

Finally, the corner contribution can be obtained when we subtract the edge and bulk parts,

$$
\begin{aligned}
\frac{\partial \Omega}{\partial \gamma} & -\frac{\partial \Omega_{\text {bulk }}}{\partial \gamma}-\frac{\partial \Omega_{\text {edge }}}{\partial \gamma} \\
= & \left\langle C^{\dagger} C\right\rangle^{\text {open }}-\left\langle C^{\dagger} C\right\rangle^{y \text {-per }}-\left\langle C^{\dagger} C\right\rangle^{x \text {-per }}+\left\langle C^{\dagger} C\right\rangle^{\text {per }} \\
= & \frac{\partial \Omega_{\text {corner }}}{\partial \gamma}=\frac{\partial \omega_{\text {corner }}}{\partial \gamma} .
\end{aligned}
$$

Calculating then the correlation functions from Eq. (9) for the open, fully periodic, $x$-periodic, and $y$-periodic boundary functions, we can obtain the different contributions in Eq. (2).

To sum up, using Eq. (8) with $\rho$ and $\sigma$ taking the lattice indices of Eq. (9), one can obtain the derivatives with respect 


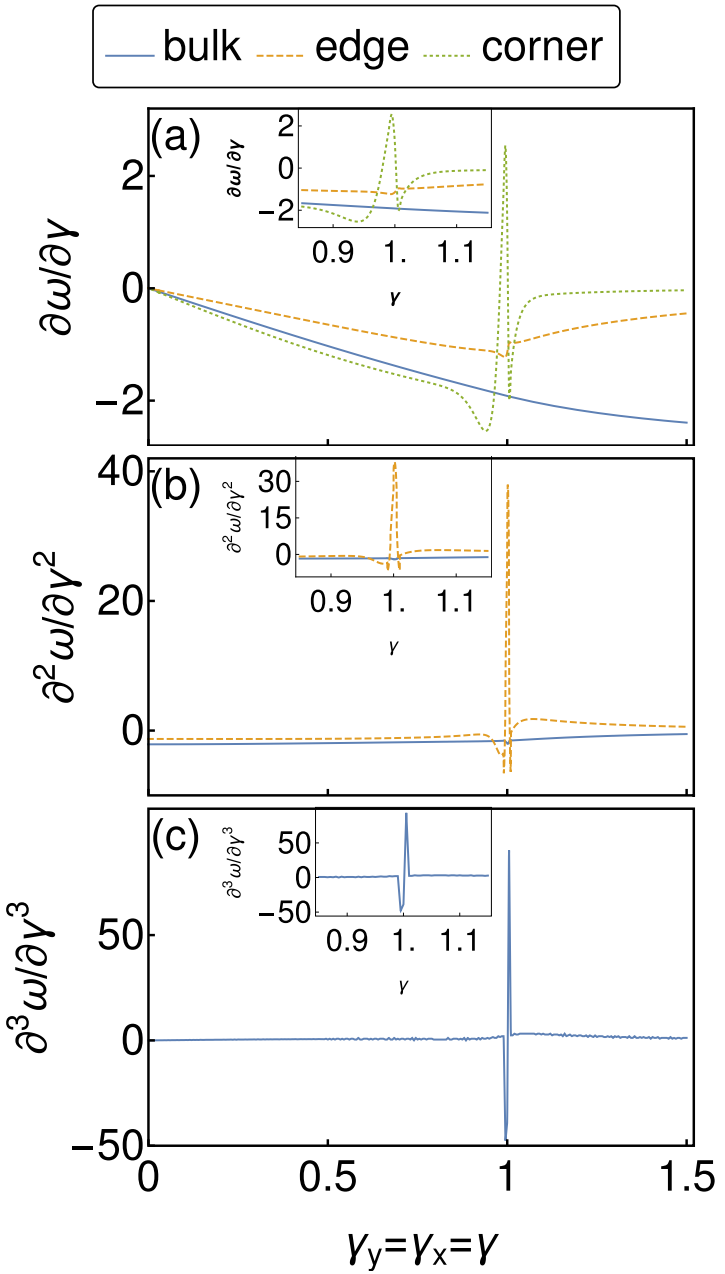

FIG. 4. (a) First, (b) second, and (c) third derivatives of the grand potential with respect to $\gamma_{x}=\gamma_{y}=\gamma$ (path I in Fig. 1) for the bulk (solid blue lines), edge (dashed yellow lines), and corner (dotted green lines) contributions. We fix $\lambda_{x}=\lambda_{y}=1$. The lattices have $40 \times 40$ unit cells. Inset: Zoom-in on the region of the phase transition.

to $\gamma$ of the different contributions to the grand potential in Eq. (2), using the correlation functions with the boundary conditions described in Eqs. (10), (14), and (15), which follow the subtraction scheme in Fig. 3.

\section{TRIVIAL-TO-QUADRUPOLAR PHASE TRANSITION}

We start by analyzing the transition from the trivial to the quadrupolar phase, path $\mathrm{I}$ in Fig. 1. The derivatives of the different contributions to the grand potential are shown in Fig. 4. For these simulations, we take the intercell hopping parameters to be $\lambda_{x}=\lambda_{y}=1$ in a lattice of $40 \times 40$ unit cells and we use interpolation of the correlation functions, such that the higher derivatives can be calculated.

In Fig. 4(a), we see that the first derivative of $\omega$ diverges for the corner contribution, while to spot the divergences of the grand potential for the edge and bulk we have to go to the second [Fig. 4(b)] and third [Fig. 4(c)] derivatives, respectively. This indicates that we can use the thermodynamic approach to identify this TQPT: the bulk exhibits a third-order phase transition, the edge a second-order phase transition, and the corner a first-order one. The main point is that the presence of the SPMs signals a discontinuity in the corresponding codimension, which is not captured when considering only the bulk free energy. These results are supported by the finite-size scaling reported in Appendix A [46].

We can further understand this behavior by analyzing the critical exponents using Josephson's hyperscaling relation.

\section{A. Josephson's hyperscaling relation}

For quantum phase transitions, the canonical critical exponent $\alpha$ determines how the grand potential scales with the reduced phase transition parameter $t$,

$$
\Omega\left(t_{-}\right)-\Omega\left(t_{+}\right) \propto|t|^{2-\alpha},
$$

where $t_{+}\left(_{-}\right)$indicates that we are approaching $t=0$ from positive (negative) values of $t$. An $n$-order derivative of $\Omega$ will scale with $t^{2-\alpha-n}$. Since $\mathrm{t} \rightarrow 0$ at the phase transition, the derivatives will start to diverge for large enough values of $n$. This implies that $2-\alpha$ is the order of the phase transition because a derivative of order higher than $2-\alpha$ will diverge.

We can determine this critical exponent, and therefore the order of the phase transition, using the Josephson hyperscaling relation $[36,47]$

$$
2-\alpha=v(d+z)
$$

where $d$ is the dimension of the system and the critical exponents $z$ and $v$ are determined by the way in which the bandgap $\Delta$ closes:

$$
\begin{aligned}
& \Delta(\mathbf{p}, t=0) \propto|\mathbf{p}|^{z}, \\
& \Delta(\mathbf{p}=0, t) \propto|t|^{v z} .
\end{aligned}
$$

Here, $p=k-K$ is the momentum relative to point $K$, at which the gap closes.

For fully periodic boundary conditions, $\lambda_{x}=\lambda_{y}=1$ and $\gamma_{x}=\gamma_{y}=\gamma$, the dispersion relation reads [6]

$$
\varepsilon_{ \pm}(\mathbf{k})= \pm \sqrt{2+2 \gamma^{2}+2 \gamma\left[\cos \left(k_{x}\right)+\cos \left(k_{y}\right)\right]}
$$

The reduced phase transition parameter takes the form $t=$ $\left(\gamma_{c}-\gamma\right) / \gamma_{c}$, where $\gamma_{c}$ is the value of the tuning parameter at which the gap closes. For this dispersion, the gap closing occurs at the $\Gamma$ point when $\gamma_{c}=-1$ and at $\mathbf{K}_{+}=( \pm \pi, \pm \pi)$ when $\gamma_{c}=1$. Then we can obtain the behavior of the gap near the phase transition and determine the critical exponents using Eq. (18).

$$
\begin{aligned}
& \text { For } \gamma_{c}=1 \text { and } \mathbf{k}=\mathbf{K}_{+}+\mathbf{p}, \\
& \qquad \Delta(\mathbf{p}, \gamma=1) \approx 2 \sqrt{2}|\mathbf{p}|,
\end{aligned}
$$

which implies that $z=1$ for this transition.

For $\mathbf{k}=\mathbf{K}_{+}$and $\gamma=(1-t)$,

$$
\Delta(\mathbf{k}= \pm \pi, t)=2 \sqrt{2}|t|,
$$

such that $v=1$ for this transition. The results are similar for $\gamma_{c}=-1$.

Then the Josephson hyperscaling relation implies that

$$
2-\alpha=d+1 \text {. }
$$


The factor $2-\alpha$ denotes how the grand potential scales with $\gamma$ at the phase transition. Therefore, this relation predicts that the transition should be third order for the bulk $(d=2)$, which was also obtained from the correlation function. In order to interpret our numerical results, we associate the phase transition in the bulk-related to the bulk-bandgap closingwith the one at the boundary-the appearance/disappearance of the SPM. If we assume that they have the same critical exponents $z$ and $v$, the only difference in the order of the phase transition should be attributed to the different dimension of each contribution. This reasoning works well for systems that exhibit a bulk-boundary correspondence [20,21], but it breaks down when the correspondence is not present [22]. Using now that the bulk is $2 \mathrm{D}$, the edge $1 \mathrm{D}$, and the corner OD in Eq. (22), we find that the corner has a first-order phase transition and the edge a second-order one, as we obtained from the correlation-function method.

\section{FIXED $\gamma_{x}$ PHASE TRANSITIONS}

We now apply the same method to investigate two other phase transitions that can be tuned in this system: the one between the dipolar and the quadrupolar phase (obtained by fixing $\gamma_{x}=0.5$ and varying $\gamma_{y}$; path II in Fig. 1) and the transition between the trivial and the dipolar phase (obtained by fixing $\gamma_{x}=1.5$ and varying $\gamma_{y}$; path III in Fig. 1).

For fixed $\gamma_{x}$, the parameter that tunes the phase transition is $\gamma_{y}$. Thus, instead of deriving Hamiltonian (1) with respect to both $\gamma_{x}$ and $\gamma_{y}$ as in Eq. (9), we derive it only with respect to $\gamma_{y}$ :

$$
\frac{\partial \Omega}{\partial \gamma_{y}}=\sum_{\mathbf{R}}\left\langle c_{\mathbf{R}, 1}^{\dagger} c_{\mathbf{R}, 4}-c_{\mathbf{R}, 2}^{\dagger} c_{\mathbf{R}, 3}+\text { H.c. }\right\rangle \text {. }
$$

We can obtain the different contributions by replacing this correlation function in Eqs. (10), (14), and (15). In Fig. 5, we plot the singular derivatives of each contribution, namely, we plot the first derivative of the corner contribution, the second one for the edge, and the third one for the bulk for each of the different phase transitions, in order to compare them.

Inspection of Figs. 5(a) and 5(b) indicates that the correlation functions exhibit discontinuities at both edge and corner for the dipolar-to-quadrupolar phase transition (dashed blue line), of about half the amplitude of the discontinuities of the transition between the trivial and the quadrupolar states. Also, there is no signal of phase transition for any component in the trivial-to-dipole transition (dotted green line) or for the bulk component in the dipolar-to-quadrupolar phase transition (dashed blue line).

To better understand why there is no response in the grand potential for these transitions, we analyze how the spectrum of the system changes along these phase transitions. In Fig. 6, we display the spectrum for $\gamma_{x}=0.5$ [Figs. 6(a)-6(d)] and $\gamma_{x}=1.5$ [Figs. 6(e)-6(h)], while we vary $\gamma_{y}$. For the dipolarto-quadrupolar transition, there is a gap closing only at the $x$ edge [Fig. 6(d)] and not at the $y$ edge [Fig. 6(c)], whereas the bulk (which has periodic boundary conditions in both directions) does not present a gap closing [Fig 6(b)]. This seems to explain why there is no signature of phase transition at the bulk while the edge and corner contributions show
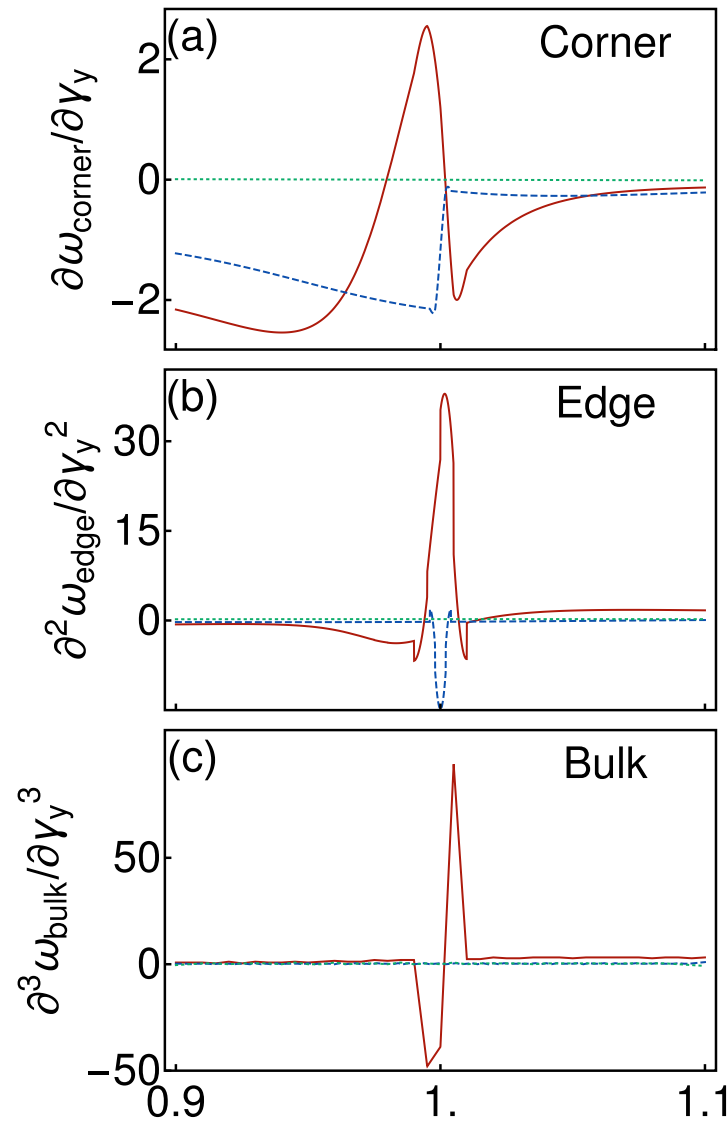

FIG. 5. (a) First derivative of the corner contribution, (b) second derivative of the edge contribution, and (c) third derivative of the bulk contribution to the grand potential with respect to $\gamma_{y}$. We consider the following three transitions: trivial to quadrupolar $\left(\gamma_{x}=\gamma_{y}=\gamma\right.$; path I in Fig. 1; solid red line), dipolar to quadrupolar $\left(\gamma_{x}=0.5\right.$; path II in Fig. 1; dashed blue line), and trivial to dipolar $\left(\gamma_{x}=1.5\right.$; path III in Fig. 1; dotted green line). The lattices have $40 \times 40$ unit cells.

discontinuities of nearly half the amplitude of the ones in the trivial-to-quadrupolar phase transition. For $\gamma_{x}=1.5$, none of the spectra shows a gap closing [Figs. 6(e) and 6(f)]. Although there is no gap closing, the wave functions change across the phases (see Fig. 7, where we plot the wave functions of the highest-energy level in the valence band for each phase). We observe that for the dipolar phase with $\gamma_{x}=1.5$ and $\gamma_{y}=0.5$, the wave function for the $x$-periodic boundary conditions is identical to the one for the same boundary condition in the quadrupolar phase, so it is localized in one of the $y$ edges and delocalized along $x$ (see Fig. 7). However, for $y$-periodic boundary conditions, the wave function is similar to the one in the trivial phase, which is delocalized (see Fig. 7). These features are captured in the polarization.

The polarization invariant changes across this phase transition without a gap closing because the symmetry that protects the polarization is related to inversion symmetry, while the gap closing occurs only for systems with $C_{4}$ symmetry [18]. The change in the polarization is associated with a nested Wilson loop [6]. However, the grand potential is not sensitive to the change in this invariant, as it is not sensitive to the presence or absence of inversion symmetry. 

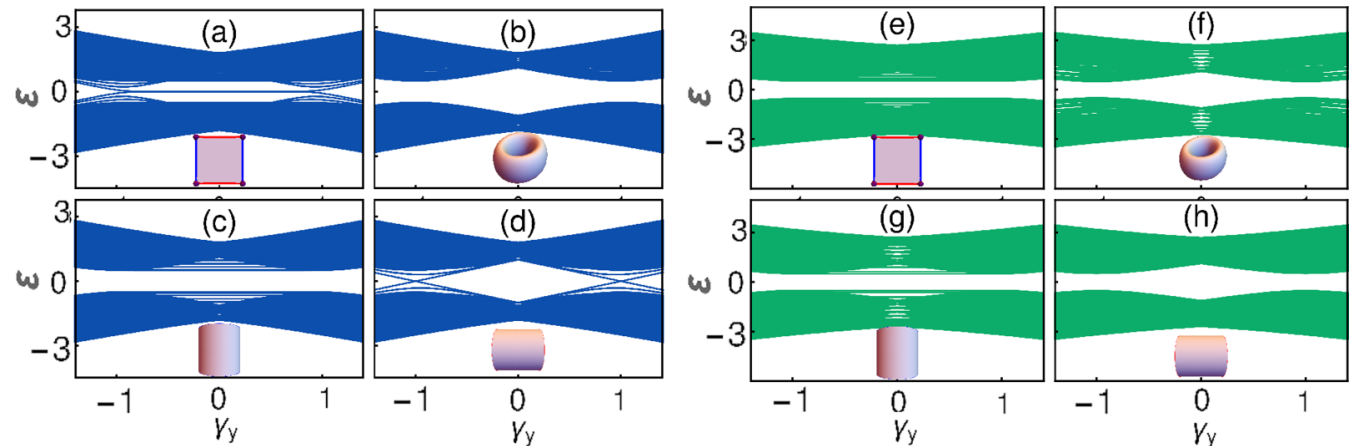

FIG. 6. Spectrum as a function of $\gamma_{y}$ for $\gamma_{x}=0.5$ (path II in Fig. 1) when the system has (a) open boundary conditions, (b) fully periodic boundary conditions, (c) $x$-periodic boundary conditions, and (d) $y$-periodic boundary conditions and for $\gamma_{x}=1.5$ (path III in Fig. 1) when the system has (e) open boundary conditions, (f) fully periodic boundary conditions, (g) $x$-periodic boundary conditions, and (h) $y$-periodic boundary conditions. In comparison with Fig. 2, we observe that the spectra for $x$-periodic and $y$-periodic boundary conditions are now different due to the absence of $C_{4}$ symmetry. The lattices have $20 \times 20$ unit cells.

The same occurs for the phase transition from the trivial to the dipolar phase. In this case, the corner, edge, and bulk contributions are all flat (dotted green lines in Fig. 5) and do not exhibit any sign of a phase transition. This behavior should be contrasted with the solid red curves in Fig. 5, which perfectly describe the TQPT from the trivial to the quadrupolar phases.

This observation can be clarified in the context of the Josephson hyperscaling relation: it is impossible to define the critical exponents $z$ and $v$ because there is no actual gap closing at this phase transition. This has the quite striking implication that we are confronted with a TQPT that does not satisfy even Ehrenfest's classification, with all thermodynamical quantities varying smoothly across the phase transition. This kind of behavior is also seen in other types of phase transitions, like the Berezinskii-Kosterlitz-Thouless [48] one, which has an infinite order.

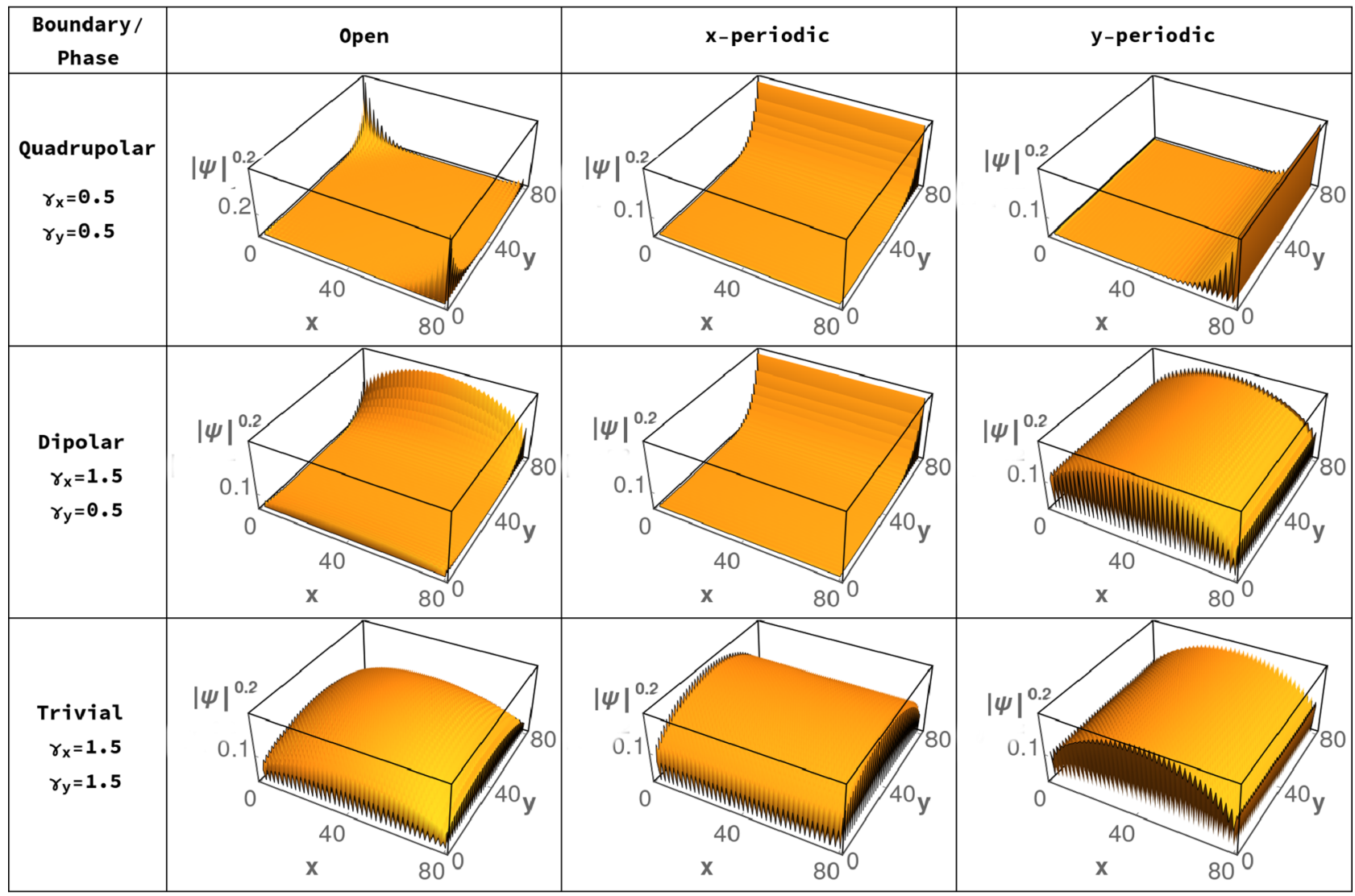

FIG. 7. Wave function for the symmetry-protected modes for several boundary conditions and for the different phases of the phase diagram shown in Fig. 1. To better visualize the localization of the wave function we actually plot $|\psi|^{0.2}$. These wave functions are evaluated for lattices with $40 \times 40$ unit cells. 


\section{WANNIER GRAND POTENTIAL}

To extract thermodynamical information for this type of phase transition, one should look at some quantities that do change across the different phase transitions. These are the Wannier centers, eigenvalues of the large Wilson loop. Consider the eigenstates of a (semi-)periodic system $\left|\psi^{m}\right\rangle(\mathbf{k})$ with band index $m$ and labeled by the momentum $\mathbf{k}$ in the periodic direction. There is a matrix $\mathbb{G}(\mathbf{k})$ associated with these eigenstates, which has the matrix elements

$$
\mathbb{G}^{m n}(\mathbf{k})=\left\langle\psi^{m}(\mathbf{k}+\Delta \mathbf{k}) \mid \psi^{n}(\mathbf{k})\right\rangle,
$$

where $m$ and $n$ are occupied bands and $|\Delta \mathbf{k}|=2 \pi / L$. $\mathbb{G}$ is called the Wilson loop element [49]. We can then define the large Wilson loop as the successive multiplication of these elements,

$$
\mathbb{W}_{\mathcal{C}, \mathbf{k}}=\mathbb{G}(\mathbf{k}-\Delta \mathbf{k}) \ldots \mathbb{G}(\mathbf{k}+\Delta \mathbf{k}) \mathbb{G}(\mathbf{k}),
$$

where $\mathcal{C}$ denotes a closed path in the first Brillouin zone that goes from $\mathbf{k}$ to $\mathbf{k}$. $\mathbb{W}$ is a unitary matrix, such that its eigenvalues are of the form $e^{i 2 \pi \zeta}$; due to topology [6,31], these eigenvalues always come in complex conjugated pairs. As a consequence, the Wannier centers $\zeta$ always come in pairs of positive and negative values. The Wilson loop can be written in terms of an effective Hamiltonian, the so-called Wannier Hamiltonian $H_{\mathrm{WC}}$,

$$
\mathbb{W}_{\mathcal{C}, \mathbf{k}}=e^{\imath 2 \pi\left[H_{\mathrm{WC}}(\mathbf{k})+\mu\right]},
$$

such that its eigenvalues are the Wannier centers (at least mod 1) and yield the Wannier spectrum. There is a gauge degree of freedom in the definition of this Hamiltonian, as we can add a term proportional to the identity to it without changing the Wilson loop. This term can be interpreted as an effective Wannier chemical potential [18].

One can, in principle, use this effective Hamiltonian to obtain these Wannier bands and derive an effective grand potential from it, the Wannier grand potential $\Phi_{W}$. To test whether one can see a gap closing or any signature of the phase transitions analyzed here, we show in Fig. 8 the Wannier spectrum for this transition for both the fully periodic and the $x$-periodic Hamiltonian, considering a path $\mathcal{C}$ that goes from $k_{x}=-\pi$ to $k_{x}=\pi$. There are clear signatures in the Wannier spectrum of all the phase transitions, either a gap closing (or the appearance of "horns" in the trivial-to-quadrupolar phase transition) for the fully periodic boundary condition or the appearance of in-gap states, which are related to a nontrivial polarization, for $x$-periodic boundary conditions. Since the exact form of the Wannier Hamiltonian in terms of operators is unknown, we are unable to identify which correlation functions are related to the derivative of $\Phi_{W}$. However, as argued in Refs. [6,18,50], the Wannier Hamiltonian of Eq. (26) is adiabatically connected to the edge Hamiltonian, with the Wannier chemical potential being related to the filling of the edge levels. In this way, the thermodynamics extracted from a Wannier Hamiltonian should correspond to the thermodynamics of a system with a $1 \mathrm{D}$ bulk and a OD boundary. In this case, we assume a form for $\Phi_{W}$ that is composed of a bulk $\phi_{\text {bulk }}$ and edge $\phi_{\text {edge }}$ contribution as

$$
\Phi_{W}=\phi_{\text {bulk }} L+\phi_{\text {edge }},
$$
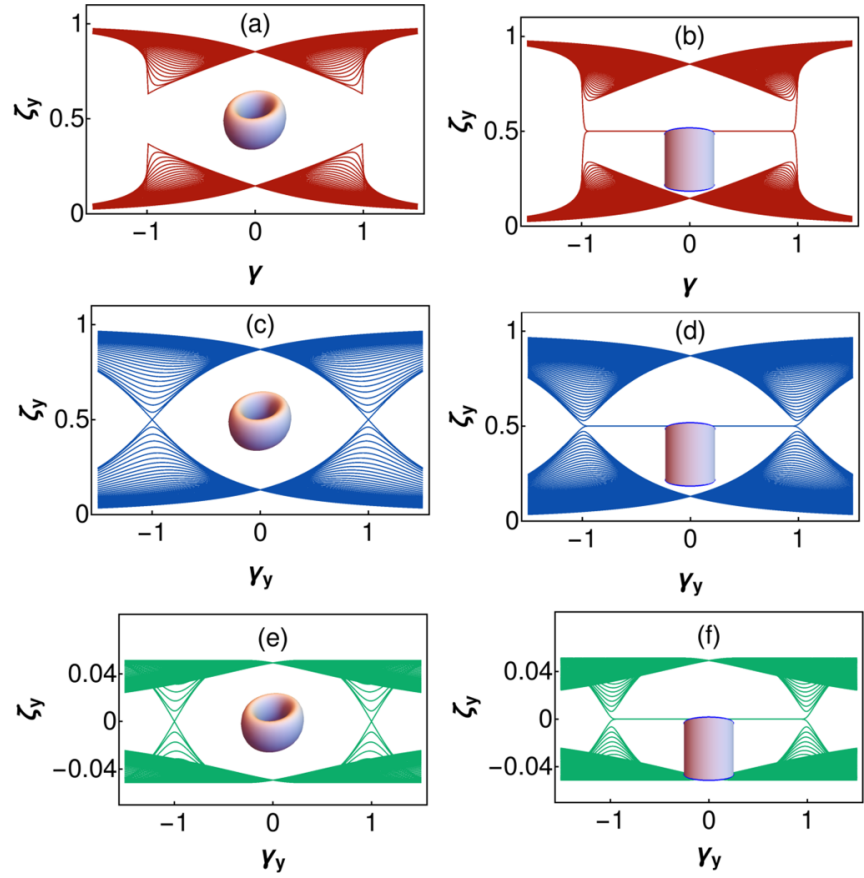

FIG. 8. Wannier spectrum for the trivial-to-quadrupolar phase transition (path I in Fig. 1) for (a) fully periodic boundary conditions and (b) $x$-periodic boundary conditions; for the dipolar-toquadrupolar phase transition (path II in Fig. 1) for (c) fully periodic boundary conditions and (d) $x$-periodic boundary conditions; and for the trivial-to-dipolar phase transition (path III in Fig. 1) for (e) fully periodic boundary conditions and (f) $x$-periodic boundary conditions. We see a signature of the phase transition in all the plots. We use a system with 100 unit cells in the nonperiodic direction and $100 k$ points to calculate the Wilson loops. Note that in (a)-(d) we choose a different gauge in relation to the one used in (e) and (f) to make the occurrence of a phase transition more evident.

such that we can obtain both contributions by

$$
\phi_{\text {bulk }}=\frac{\Phi_{W}^{\text {per }}}{L}
$$

and

$$
\phi_{\text {edge }}=\Phi_{W}^{\mathrm{x}-\text { per }}-\Phi_{W}^{\mathrm{per}} .
$$

For free fermions at zero temperature, the grand potential defined in Eq. (3) is given by

$$
\begin{aligned}
\Omega & =-\lim _{T \rightarrow 0} \frac{1}{\beta} \sum_{m} \ln \left[1+e^{-\beta\left(\varepsilon_{m}-\mu\right)}\right] \\
& =\sum_{\varepsilon_{m} \leqslant \mu}\left(\varepsilon_{m}-\mu\right),
\end{aligned}
$$

where $m$ are all available states.

In an analogous way, the Wannier grand potential at $T=0$ is defined by

$$
\Phi_{W}=\sum_{\zeta \leqslant \mu} \zeta,
$$

where now the chemical potential $\mu$ should be set to 0.5 for the trivial-to-quadrupolar or dipolar-to-quadrupolar phase transition, as is evident in the spectra in Fig. 8. This definition at 

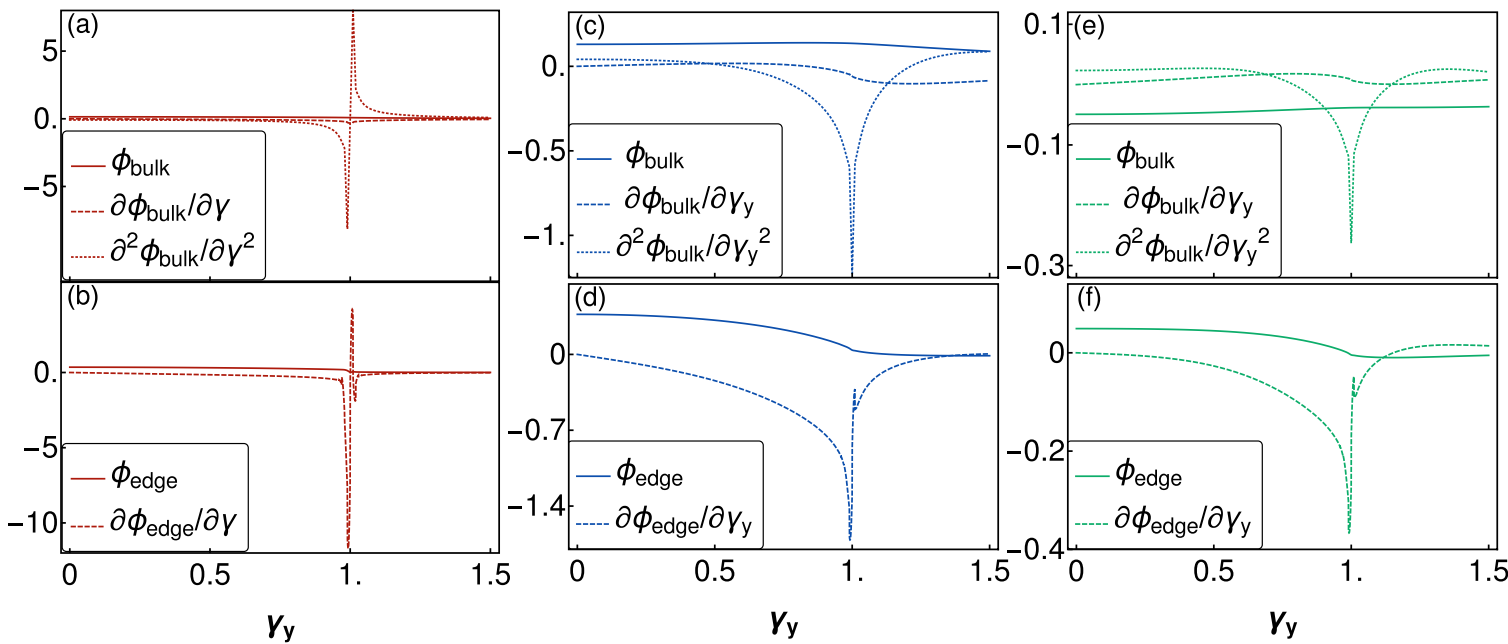

FIG. 9. Wannier grand potential (solid lines) and its first (dashed lines) and second (dotted lines) derivatives for the (a) bulk and (b) edge contributions along the trivial-to-quadrupolar phase transition (path I in Fig 1; red lines). (c) Bulk and (d) edge contributions along the dipolar-to-quadrupolar phase transition (path II in Fig 1; blue lines). (e) Bulk and (f) edge contributions along the trivial-to-dipolar phase transition (path III in Fig 1; green lines). We use a system with 200 unit cells in the nonperiodic direction and $100 k$ points to calculate the Wilson loops.

zero temperature will be associated with the polarization, with the difference that the Wannier grand potential is uniquely defined by the filling of the edge Hamiltonian. Note that Eq. (30) provides a more general description of the system at finite temperatures if the limit $T \rightarrow 0$ is not taken.

We calculate these quantities using the Wannier spectra and the results are presented in Fig. 9. The phase transitions for the 1D bulk are second order, while for the OD edge these transitions are first order. It is interesting to note that even in the absence of an actual gap closing in the trivial-toquadrupolar phase transition, the Wannier grand potential is sensitive to the phase transition.

As the Wannier gap closes with the same critical exponents as the energy gap of Eq. (18) (see Appendix B), these two transitions belong to the same universality class. We see that in this case, these phase transitions indeed satisfy Josephson's hyperscaling relation given in Eq. (22) for a 1D bulk and a OD edge. The hyperscaling relation together with the scaling of the Wannier grand potential reinforces the identification of the Wannier Hamiltonian with the edge Hamiltonian.

The fact that we can identify all the phase transitions using the Wannier grand potential indicates that the Wilson loop carries topological information that is not contained in the usual band structure, which is already suggested by the absence of any gap closing or appearance of in-gap states in the spectra in Fig. 6. However, assuming that the Wannier centers are indeed adiabatically connected to the edge
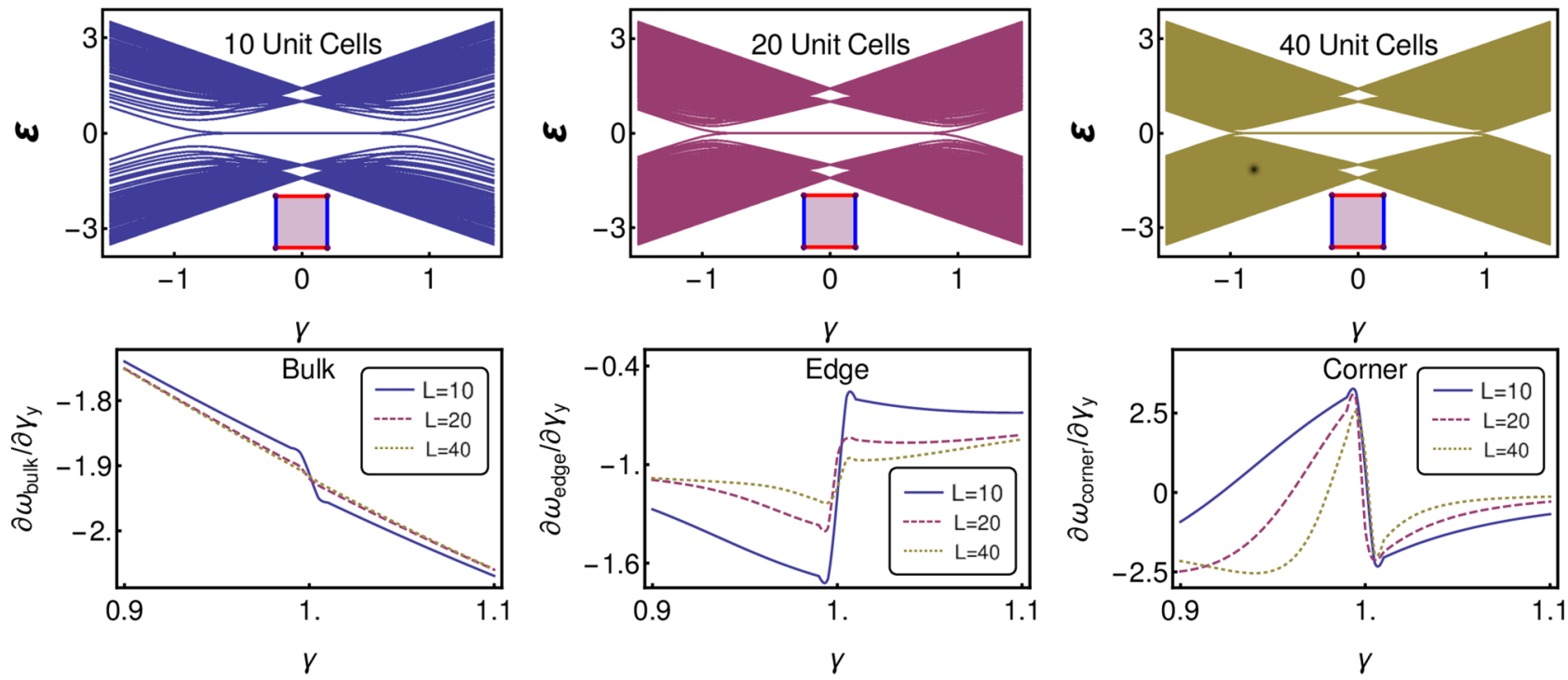

FIG. 10. Finite-size effects. Spectrum and potential contributions for systems with $10 \times 10$ (solid blue lines), $20 \times 20$ (dashed purple lines), and $40 \times 40$ (dotted yellow lines) unit cells for the trivial-to-quadrupolar phase transition (path I in Fig. 1). 
spectrum, it is quite surprising that they yield a signature of the phase transition, while the actual spectrum, which contains the edge levels, does not provide this information. A possible answer to this question is that, as pointed out in Ref. [50], the density matrix obtained from the Wannier Hamiltonian is basically a purification of the density matrix obtained from the physical Hamiltonian. In this way, it might be possible to obtain the Wannier grand potential after a partial trace in the grand potential over the degrees of freedom related to the periodic direction that compose the large Wilson loop, but this is beyond the scope of this work.

\section{CONCLUSIONS}

We have extended the formalism used in Refs. [19-22,28] to investigate HOTIs, where the bulk-boundary correspondence relates the closure of the bandgap to the zero modes that occur at the corners of the system. We have numerically calculated the spectrum of Hamiltonian (1), which was proposed in Ref. [6], to identify the discontinuities in the derivative of the grand potential and elucidate the order of the TQPT. We found that for the trivial-to-quadrupolar phase transition, the bulk exhibits a third-order, the edge a second-order, and the corner a first-order phase transition, in agreement with Josephson's hyperscaling relation.

However, some of the TQPTs are not captured by the thermodynamic potential description, since they showed no discontinuities at transitions between states with different polarizations. This happens due to the absence of a bandgap closing at these phase transitions. We then constructed a Wannier grand potential using the Wannier centers obtained via the Wilson loop, as the Wannier bands are expected to track the TQPT in situations where there is no energy gap closing.

The Wannier grand potential presents critical exponents that have, according to the Josephson hyperscaling relation, the same universality class as a system with a $1 \mathrm{D}$ bulk and a OD edge. This, together with the scaling of the Wannier grand potential, confirms the identification of the Wannier Hamiltonian with the edge Hamiltonian.

Deeper understandings of this Wannier grand potential and its implication for evaluations of the entanglement entropy or Otto heat cycles, as well as a more complete comprehension of how one can relate the actual grand potential to the Wannier grand potential, are interesting topics for future work.

\section{ACKNOWLEDGMENTS}

We thank Carlo Beenakker and Tom O' Brien for the access to the Maris cluster in Leiden, where some of the simulations were performed. We are grateful to Jette van den Broeke and Peter Cats for fruitful discussions. We would like to thank
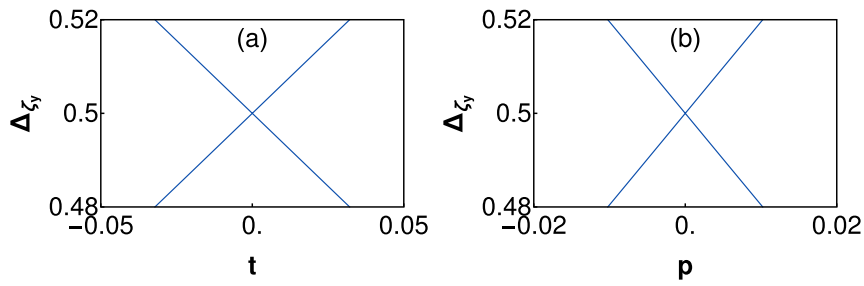

FIG. 11. The Wannier gap closes linearly with both (a) $t=\gamma_{y}-$ $1\left(k_{x}=\pi\right)$ and (b) $p=\left(k_{x}-\pi\right) / \pi\left(\gamma_{y}=1.0\right)$.

Raquel Queiroz and Sander Kooi for bringing Ref. [18] to our attention. This work was mainly developed during the period when R.A. was a Ph.D. student visitor at Utrecht University via the Delta Institute for Theoretical Physics (Delta ITP) consortium, a program of the Netherlands Organization for Scientific Research (NWO), which is funded by the Dutch Ministry of Education, Culture and Science. R.A. acknowledges funding from the Brazilian National Council for Scientific and Technological Development (CNPq) and the Brazilian Coordination for the Improvement of Higher Education Personnel (CAPES). S.N.K. was financed by NWO under Grant No. 16PR3245.

\section{APPENDIX A: FINITE-SIZE EFFECTS}

Here we comment on the main problem that arises due to finite-size effects, that is, the hybridization between corner modes when the system size is too small and the gap does not close at $|\gamma|=1$. In this case, the corner modes hybridize and move away from 0 , while the bulk gap remains open. As the correlation function is calculated from the spectrum, we can use the gap closing as a guideline to verify whether the system is large enough to reveal the phase transition. In Fig. 10, we show the finite-size effects for the first derivative of the bulk, edge, and corner contributions for different system sizes. Although a small kink appears close to $\gamma=1$ for the bulk and edge first derivative, it becomes smaller and broader when we increase the system size, indicating a finite-size effect. In comparison, the divergence of the corner contribution becomes narrower as we increase the system size, indicating that it is indeed related to a discontinuity.

\section{APPENDIX B: BEHAVIOR OF THE WANNIER GAP}

Here, we show that the Wannier gap also closes linearly in both $\mathbf{p}$ and $t$, such that $v=1$ and $z=1$, as commented in Sec. VI. In Fig. 11, we show that the Wannier gap closes linearly at the TQPT for both $t=\gamma_{y}-1\left(k_{x}=\pi\right)$ and $p=$ $\left(k_{x}-\pi\right) / \pi\left(\gamma_{y}=1.0\right)$ for the quadrupolar-to-dipolar phase transition. The other phase transitions show the same critical exponents.
[1] A. Altland and M. R. Zirnbauer, Phys. Rev. B 55, 1142 (1997).

[2] A. P. Schnyder, S. Ryu, A. Furusaki, and A. W. W. Ludwig, Phys. Rev. B 78, 195125 (2008).
[3] A. Kitaev, in AIP Conference Proceedings, Vol. 1134 (American Institute of Physics, Melville, NY, 2009), pp. 22-30.

[4] L. Fu, Phys. Rev. Lett. 106, 106802 (2011). 
[5] R.-J. Slager, A. Mesaros, V. Juričić, and J. Zaanen, Nat. Phys. 9, 98 (2013).

[6] W. A. Benalcazar, B. A. Bernevig, and T. L. Hughes, Science 357, 61 (2017); Phys. Rev. B 96, 245115 (2017).

[7] F. Schindler, Z. Wang, M. G. Vergniory, A. M. Cook, A. Murani, S. Sengupta, A. Y. Kasumov, R. Deblock, S. Jeon, I. Drozdov et al., Nat. Phys. 14, 918 (2018).

[8] S. Imhof, C. Berger, F. Bayer, J. Brehm, L. W. Molenkamp, T. Kiessling, F. Schindler, C. H. Lee, M. Greiter, T. Neupert et al., Nat. Phys. 14, 925 (2018).

[9] M. Serra-Garcia, R. Süsstrunk, and S. D. Huber, Phys. Rev. B 99, 020304(R) (2019).

[10] X. Ni, M. Weiner, A. Alù, and A. B. Khanikaev, Nat. Mater. 18, 113 (2019).

[11] H. Xue, Y. Yang, F. Gao, Y. Chong, and B. Zhang, Nat. Mater. 18, 108 (2019).

[12] S. Kempkes, M. Slot, J. van den Broeke, P. Capiod, W. Benalcazar, D. Vanmaekelbergh, D. Bercioux, I. Swart, and C. M. Smith, Nat. Mater. 18, 1292 (2019).

[13] A. Matsugatani and H. Watanabe, Phys. Rev. B 98, 205129 (2018).

[14] M. Ezawa, Phys. Rev. B 98, 201402(R) (2018).

[15] D. Călugăru, V. Juričić, and B. Roy, Phys. Rev. B 99, 041301(R) (2019).

[16] A. Agarwala, V. Juričić, and B. Roy, Phys. Rev. Res. 2, 012067 (2020).

[17] L. Trifunovic and P. W. Brouwer, Phys. Rev. X 9, 011012 (2019).

[18] E. Khalaf, W. A. Benalcazar, T. L. Hughes, and R. Queiroz, arXiv:1908.00011.

[19] A. Quelle, E. Cobanera, and C. M. Smith, Phys. Rev. B 94, 075133 (2016).

[20] S. Kempkes, A. Quelle, and C. M. Smith, Sci. Rep. 6, 38530 (2016).

[21] J. van den Broeke, S. Kempkes, A. Quelle, X. Wang, J. Paglione, and C. M. Smith, arXiv:1803.03553.

[22] P. Cats, A. Quelle, O. Viyuela, M. Martin-Delgado, and C. M. Smith, Phys. Rev. B 97, 121106(R) (2018).

[23] T. L. Hill, Thermodynamics of Small Systems (Courier Corp., North Chelmsford, MA, 1994).

[24] R. V. Chamberlin, Phys. Rev. Lett. 82, 2520 (1999).

[25] R. V. Chamberlin, Nature 408, 337 (2000).

[26] R. V. Chamberlin, Entropy 17, 52 (2015).

[27] I. Latella, A. Pérez-Madrid, A. Campa, L. Casetti, and S. Ruffo, Phys. Rev. Lett. 114, 230601 (2015).
[28] E. Yunt, M. Fadaie, Ö. E. Müstecaplioglu, and C. M. Smith, arXiv:2003.08836.

[29] R. Resta, Ferroelectrics 151, 49 (1994).

[30] N. A. Spaldin, J. Solid State Chem. 195, 2 (2012).

[31] T. Neupert and F. Schindler, in Topological Matter (Springer, Berlin, 2017).

[32] W. Chen, M. Sigrist, and A. P. Schnyder, J. Phys.: Condens. Matter 28, 365501 (2016).

[33] W. Chen, Phys. Rev. B 97, 115130 (2018).

[34] W. Chen and A. P. Schnyder, New J. Phys. 21, 073003 (2019).

[35] P. Molignini, W. Chen, and R. Chitra, Phys. Rev. B 101, 165106 (2020).

[36] P. Molignini, R. Chitra, and W. Chen, Europhys. Lett. 128, 36001 (2020); M. A. Continentino, Quantum Scaling in ManyBody Systems (Cambridge University Press, Cambridge, UK, 2017).

[37] M. A. Continentino, Physica B: Condens. Matter 505, A1 (2017).

[38] M. A. Griffith and M. A. Continentino, Phys. Rev. E 97, 012107 (2018).

[39] S. Rufo, N. Lopes, M. A. Continentino, and M. A. R. Griffith, Phys. Rev. B 100, 195432 (2019).

[40] Z.-Q. Wang, G.-Y. Zhu, and G.-M. Zhang, Phys. Rev. B 98, 155139 (2018).

[41] A. B. Pippard, Elements of Classical Thermodynamics (Cambridge University Press, Cambridge, UK, 1957).

[42] G. Jaeger, Arch. Hist. Exact Sci. 53, 51 (1998).

[43] Z. H. Li and D. G. Truhlar, Chem. Sci. 5, 2605 (2014).

[44] T. L. Hill, Nano Lett. 1, 273 (2001).

[45] D. Bedeaux and S. Kjelstrup, Chem. Phys. Lett. 707, 40 (2018).

[46] Note that the edge and corner contributions are still finite, even in the trivial phase. These contributions are related to finitesize effects-which often come into play when considering nonextensive quantities - and go to 0 when $\gamma$ is increased in the trivial phase.

[47] M. A. Continentino, Phys. Rep. 239, 179 (1994).

[48] J. M. Kosterlitz and D. J. Thouless, J. Phys. C 6, 1181 (1973).

[49] The actual Wilson loop element will be the unitary version of this matrix, as this matrix is unitary only in the thermodynamical limit, as discussed in [6].

[50] L. Fidkowski, T. S. Jackson, and I. Klich, Phys. Rev. Lett. 107, 036601 (2011). 